\title{
ALGEBRAIC POLYNOMIALS WITH RANDOM COEFFICIENTS
}

\author{
K. FARAHMAND \\ University of Ulster \\ Department of Mathematics \\ Jordanstown, Co. Antrim BT37 0QB, UK
}

(Received January, 2001, Revised June, 2001)

This paper provides an asymptotic value for the mathematical expected number of points of inflections of a random polynomial of the form $a_{0}(\omega)+a_{1}(\omega)\left(\begin{array}{l}n \\ 1\end{array}\right)^{1 / 2} x+a_{2}(\omega)\left(\begin{array}{l}n \\ 2\end{array}\right)^{1 / 2} x^{2}+\ldots a_{n}(\omega)\left(\begin{array}{l}n \\ n\end{array}\right)^{1 / 2} x^{n}$ when $n$ is large. The coefficients $\left\{a_{j}(\omega)\right\}_{j=0}^{n}, \omega \in \Omega$ are assumed to be a sequence of independent normally distributed random variables with means zero and variance one, each defined on a fixed probability space $(A, \Omega, \operatorname{Pr})$. A special case of dependent coefficients is also studied.

Key words: Number of Real Roots, Random Algebraic Polynomials, KacRice Formula, Random Variables, Points of Inflection.

AMS subject classifications: 60G99, 60H99.

\section{Introduction}

Recently, there has been increasing interest in the study of mathematical behavior of random algebraic polynomials of the form

$$
P_{n}(x)=\sum_{j=0}^{n}\left(\begin{array}{c}
j \\
n
\end{array}\right)^{1 / 2} a_{j}(\omega) x^{j}
$$

It is assumed that the coefficients $\left\{a_{j}(\omega)\right\}_{j=0}^{n}, \omega \in \Omega$ are a sequence of random variables defined on a fixed probability space $(A, \Omega, \operatorname{Pr})$. Motivated by their applications in physics, see for example [2] and [9], as well as, of course, mathematical and probabilistic properties, we denote the expected number of real zeros of $P_{n}(x)$ by $E N$. In [3] and [5] for the case of normal standard distribution of the coefficients, it is shown that, in the interval $(-\infty, \infty)$ and for $n$ large $E N(-\infty, \infty) \sim \sqrt{n}$. Also other properties of $P_{n}(x)$ such as the number of $K$-level crossings, that is the expected number of real roots of $P_{n}(x)=K$, or the expected number of maxima of $P_{n}(x)$ are subsequently studied in [5-7]. A comprehensive study of different types of random polynomials can be found in Bharucha-Reid and Sambandham [1]. More recent developments of the subject as well as particular results on $P_{n}(x)$ can be found in Farahmand [4]. As we can see from the latter, on account of the analytical difficulties, it has not been possible to obtain any significant results for $E N$ by relaxing the above assumptions on the distributions of the coefficients. Therefore, it is of special interest to reveal additional mathematical behavior of $P_{n}(x)$, albeit under the above assumptions of normality for the distribution of the coefficients. To this end, our main task here is to obtain 
the expected number of points of inflection of $P_{n}(x)$ by insisting on normal standard distribution for the coefficients.

The point $\left(c, P_{n}(c)\right)$ is a point of inflection of the polynomial if there exists an open interval, containing $c$, such that the second derivative of $P_{n}(x)$ satisfies either of the following inequalities

$$
P_{n}^{\prime \prime}(x)<0 \text { if } x<c \text { and } P_{n}^{\prime \prime}(x)>0 \text { if } x>c
$$

or

$$
P_{n}^{\prime \prime}(x)>0 \text { if } x<c \text { and } P_{n}^{\prime \prime}(x)<0 \text { if } x>c .
$$

It is known that, since $P_{n}(x)$ is differentiable on any open interval and if $\left(c, P_{n}(c)\right)$ is a point of inflection of $P_{n}(x)$, then $P_{n}^{\prime \prime}(c)=0$. We note, however, that even if $P_{n}^{\prime \prime}(c)=0$ then $P_{n}(x)$ may not be a stationary point at $c$. For this to occur, $P_{n}(c)=0, P_{n}^{\prime}(c)=0$ and $P_{n}^{\prime \prime}(c)=0$ must necessarily hold. But the set of such parameter $c$ has measure zero. Therefore it is sufficient to obtain the expected number of real zeros of $P_{n}^{\prime \prime}(x)$. To this end, we use the Kac-Rice formula [8] and [10] for $P_{n}^{\prime \prime}(x)$. Let $I_{n}(a, b)$ be the number of points of inflection of $P_{n}(x)$ in the interval $(a, b)$ and $E I_{n}(a, b)$ its expected value. Then from the Kac-Rice formula it is known that

$$
E I_{n}(a, b)=\frac{1}{\pi} \int_{a}^{b} \frac{\Delta}{A^{2}} d x
$$

where

$$
\begin{gathered}
A^{2}=\operatorname{var}\left(P_{n}^{\prime \prime}(x)\right), B^{2}=\operatorname{var}\left(P_{n}^{\prime \prime \prime}(x)\right) \\
C=\operatorname{cov}\left(P_{n}^{\prime \prime}(x), P_{n}^{\prime \prime \prime}(x)\right) \text { and } \Delta^{2}=A^{2} B^{2}-C^{2} .
\end{gathered}
$$

We prove

Theorem 1: If the coefficients of $P_{n}(x)$ are independent normally distributed with mean zero and variance one, then for any $T>0$ the expected number of points of inflection of $P_{n}(x)$ satisfies

$$
E I_{n}(-T, T) \sim \frac{2 \sqrt{n-2}}{\pi} \arctan (T)
$$

We conclude by showing our method can yield the expected number of real zeros of $P_{n}(x)$ for a special case of dependent coefficients.

\section{Moments and Primary Analysis}

In order to apply the Kac-Rice formula (1.1) to $P_{n}^{\prime \prime}(x)$ and obtain the expected number of points of inflection of $P_{n}(x)$, we need to obtain the variances of $P_{n}^{\prime \prime}(x)$ and $P_{n}^{\prime \prime \prime}(x)$ as well as their covariances. To this end, we obtain the following identities, the first of which is well known and the remaining are obtained by consequent differentiation of the previous one multiplied by $x$.

$$
\sum_{j=0}^{n}\left(\begin{array}{c}
n \\
j
\end{array}\right) x^{j}=(x+1)^{n}
$$




$$
\begin{aligned}
& \sum_{j=0}^{n} j\left(\begin{array}{c}
n \\
j
\end{array}\right) x^{j}=n x(x+1)^{n-1} \\
& \sum_{j=0}^{n} j^{2}\left(\begin{array}{c}
n \\
j
\end{array}\right) x^{j}=n x(x+1)^{n-1}+n(n-1) x^{2}(x+1)^{n-2} \\
& \sum_{j=0}^{n} j^{3}\left(\begin{array}{c}
n \\
j
\end{array}\right) x^{j}=n x(x+1)^{n-1}+3 n(n-1) x^{n}(x+1)^{n-2} \\
& +n(n-1)(n-2) x^{3}(x+1)^{n-3} \\
& \sum_{j=0}^{n} j^{4}\left(\begin{array}{c}
n \\
j
\end{array}\right) x^{j}=n x(x+1)^{n-1}+7 n(n-1) x^{2}(x+1)^{n-2} \\
& +6 n(n-1)(n-2) x^{3}(x+1)^{n-3}+n(n-1)(n-2)(n-3) x^{4}(x+1)^{n-4} \\
& \sum_{j=0}^{n} j^{5}\left(\begin{array}{c}
n \\
j
\end{array}\right) x^{j}=n x(x+1)^{n-1}+15 n(n-1) x^{2}(x+1)^{n-2} \\
& +25 n(n-1)(n-2) x^{3}(x+1)^{n-3}+10 n(n-1)(n-2)(n-3) x^{4}(x+1)^{n-4} \\
& +n(n-1)(n-2)(n-3)(n-4) x^{5}(x+1)^{n-5}
\end{aligned}
$$

and

$$
\begin{gathered}
\sum_{j=0}^{n} j^{6}\left(\begin{array}{c}
n \\
j
\end{array}\right) x^{j}=n x(x+1)^{n-1}+31 n(n-1) x^{2}(x+1)^{n-2} \\
+90 n(n-1)(n-2) x^{3}(x+1)^{n-3}+65 n(n-1)(n-2)(n-3) x^{4}(x+1)^{n-4} \\
+15 n(n-1)(n-2)(n-3)(n-4) x^{5}(x+1)^{n-5} \\
+n(n-1)(n-2)(n-3)(n-4)(n-5) x^{6}(x+1)^{n-6} .
\end{gathered}
$$

Now since the $j$ th coefficient of $P_{n}(x)$ has variance $\left(\begin{array}{c}n \\ j\end{array}\right)$ we can use (2.3)-(2.5) to obtain

$$
\begin{gathered}
A^{2}=\operatorname{var}\left(P_{n}^{\prime \prime}(x)\right)=\sum_{j=0}^{n} j^{2}(j-1)^{2}\left(\begin{array}{c}
n \\
j
\end{array}\right) x^{2 j-4} \\
=n(n-1)\left(x^{2}+1\right)^{n-4}\left\{2\left(x^{2}+1\right)^{2}+4(n-2) x^{2}\left(x^{2}+1\right)+(n-2)(n-3) x^{4}\right\} .
\end{gathered}
$$

Similarly using (2.3)-(2.7) yields 


$$
\begin{gathered}
B^{2}=\operatorname{var}\left(P_{n}^{\prime \prime \prime}(x)\right)=\sum_{j=0}^{n} j^{2}(j-1)^{2}(j-2)^{2}\left(\begin{array}{c}
n \\
j
\end{array}\right) x^{2 j-6} \\
=n(n-1)(n-2)\left(x^{2}+1\right)^{n-6}\left\{6\left(x^{2}+1\right)^{3}+18(n-3) x^{2}\left(x^{2}+1\right)^{2}\right. \\
\left.+9(n-3)(n-4) x^{4}\left(x^{2}+1\right)+(n-3)(n-4)(n-5) x^{6}\right\}
\end{gathered}
$$

and

$$
\begin{gathered}
C=\operatorname{cov}\left(P_{n}^{\prime \prime}(x), P_{n}^{\prime \prime \prime}(x)\right)=\sum_{j=0}^{n} j^{2}(j-1)^{2}(j-2)\left(\begin{array}{c}
n \\
j
\end{array}\right) x^{2 j-5} \\
=n(n-1)(n-2) x\left(x^{2}+1\right)^{n-5}\left\{6\left(x^{2}+1\right)^{2}+6(n-3) x^{2}\left(x^{2}+1\right)+(n-3)(n-4) x^{4}\right\} .
\end{gathered}
$$

\section{Expected Number of Points of Inflection}

Now that we have obtained the required identities for the Kac-Rice formula, we can proceed to derive the expected number of points of inflection. Although for what follows, we do not require the exact value of $\Delta^{2}$, for convenience and perhaps future use we give its value. As we will see in (3.2) and (3.3), we only need an estimate of $\Delta^{2}$ valid for all sufficiently large $n$. From (2.8)-(2.10) and simple algebra we obtain:

$$
\begin{gathered}
\Delta^{2}=n^{2}(n-1)^{2}(n-2)\left(x^{2}+1\right)^{2 n-10}\left\{12\left(x^{2}+1\right)^{5}\right. \\
+36(n-3) x^{2}\left(x^{2}+1\right)^{4}+12(n-2) x^{2}\left(x^{2}+1\right)^{4} \\
+18(n-3)(n-4) x^{4}\left(x^{2}+1\right)^{3}+6(n-2)(n-3) x^{4}\left(x^{2}+1\right)^{3} \\
+2(n-3)(n-4)(n-5) x^{6}\left(x^{2}+1\right)^{2}-18(n-2)(n-3)^{2} x^{6}\left(x^{2}+1\right)^{2} \\
+48(n-2)(n-3)(n-4) x^{6}\left(x^{2}+1\right)^{2}+4(n-2)(n-3)(n-4)(n-5) x^{8}\left(x^{2}+1\right) \\
-3(n-2)(n-3)^{2}(n-4) x^{8}\left(x^{2}+1\right)+(n-2)(n-3)^{2}(n-4)(n-5) x^{10} \\
\left.-(n-2)(n-3)^{2}(n-4)^{2} x^{10}\right\} .
\end{gathered}
$$

Now for $n$ large the leading terms in (3.1), that is the terms involved with $n^{5}$ inside \{\} , are eliminated. Therefore, we require consideration of the terms with $n^{4}$ as their leading term. Then we can easily show that for all sufficiently large $n$,

$$
\Delta^{2} \sim n^{2}(n-1)^{2}(n-2)\left(x^{2}+1\right)^{2 n-10}\left\{n^{4} x^{8}\left(x^{2}+1\right)-n^{4} x^{10}\right\}
$$




$$
\sim n^{6}(n-1)^{2}(n-2) x^{8}\left(x^{2}+1\right)^{2 n-10} .
$$

Now from the Kac-Rice formula (1.1), (2.8) and (3.2) for all sufficiently large $n$, we obtain

$$
\begin{gathered}
E I_{n}(-T, T)=\frac{1}{\pi} \int_{-T}^{T} \frac{\Delta}{A^{2}} d x \sim \frac{\sqrt{n-2}}{\pi} \int_{-T}^{T} \frac{d x}{x^{2}+1} \\
=\frac{2 \sqrt{n-2}}{\pi} \arctan (T) .
\end{gathered}
$$

This completes the proof of the theorem.

\section{Dependent Coefficients}

Here we study the effect of certain dependency of the coefficients $P_{n}(x)$. We assume that, although as before the coefficients $a_{j}$ 's are normally distributed with means zero they possess the moment matrix with $\rho_{i j}=\rho\left(\begin{array}{c}n \\ i\end{array}\right)^{1 / 2}\left(\begin{array}{c}n \\ j\end{array}\right)^{1 / 2}$ for all $i, j=0,1,2, \ldots, n$. In other words, $\left\{a_{j}\right\}_{j=0}^{n}$ is a sequence of dependent normally distributed random variables with mean zero and joint density function $\sqrt{|\Pi| /(2 \pi)^{n}} \exp \left\{-(1 / 2) \mathrm{a}^{\prime} \Pi a\right\}$, where $\Pi^{-1}$ is the moment matrix with elements $\rho_{i j}$ as above. With these assumptions, we can evaluate the moments of $P_{n}(x)$ and $P_{n}^{\prime}(x)$. First

$$
\begin{gathered}
A_{\rho}^{2}=\operatorname{var}\left(P_{n}(x)\right)=\sum_{j=0}^{n}\left(\begin{array}{c}
n \\
j
\end{array}\right) x^{2 j} E\left(a_{j}^{2}\right)+\sum_{j=0}^{n} \sum_{\substack{j=0 \\
i \neq j}}^{n}\left(\begin{array}{l}
n \\
i
\end{array}\right)^{1 / 2}\left(\begin{array}{c}
n \\
j
\end{array}\right)^{1 / 2} x^{i+j} E\left(a_{i} a_{j}\right) \\
=\rho\left(\sum_{j=0}^{n}\left(\begin{array}{c}
n \\
j
\end{array}\right) x^{j}\right)^{2} .
\end{gathered}
$$

Similarly,

$$
B_{\rho}^{2}=\operatorname{var}\left(P_{n}^{\prime}(x)\right)=\rho\left(\sum_{j=0}^{n}\left(\begin{array}{c}
n \\
j
\end{array}\right) j x^{j-1}\right)^{2}
$$

and

$$
C_{\rho}=\operatorname{cov}\left(P_{n}(x), P_{n}^{\prime}(x)\right)=\rho\left(\sum_{j=0}^{n}\left(\begin{array}{c}
n \\
j
\end{array}\right) x^{j}\right)\left(\sum_{j=0}^{n}\left(\begin{array}{c}
n \\
j
\end{array}\right) j x^{j-1}\right) .
$$

It is obvious from (4.1)-(4.3) that $\Delta_{\rho}^{2}=A_{\rho}^{2} B_{\rho}^{2}-C_{\rho}^{2}=0$ and therefore $E N=0$. This is interesting as it shows that the expected number of zeros of $P_{n}(x)$ is zero. However, the above assumptions are critical for obtaining results. Any slight derivation from our above assumptions yield terms in (4.1)-(4.3) which are not possible or difficult to evaluate analytically. 


\section{References}

[1] Bharucha-Reid, A.T. and Sambandham, M., Random Polynomials, Academic Press, New York 1986.

[2] Bogomolny, E., Bohigas, O. and Leboeuf, P., Distribution of roots of random polynomials, Phys. Rev. Lett. 68 (1992), 2726-2729.

[3] Edelman, A. and Kostlan, E., How many zeros of a random polynomial are real?, Bull. Amer. Math. Soc. 32 (1995), 1-37.

[4] Farahmand, K., Topics in Random Polynomials, Addison Wesley Longman, London 1998.

[5] Farahmand, K., On random algebraic polynomials, Proc. Amer. Math. Soc. 127 (1999), 3339-3344.

[6] Farahmand, K., On algebraic polynomials with random coefficients, Proc. Amer. Math. Soc. 129 (2001), 2763-2769.

[7] Farahmand, K. and Grigorash, A., Level crossings of random algebraic polynomials with non-identically distributed normal coefficients, Intern. J. of Appl. Math. 2 (2000), 213-221.

[8] Kac, M., On the average number of real roots of a random algebraic equations, Bull. Amer. Math. Soc. 49 (1943), 314-320.

[9] Ramponi, A., A note on the complex roots of complex random polynomials, Stats. and Prob. Lett. 44 (1999), 181-187.

[10] Rice, S.O., Mathematical theory of random noise, Bell. System Tech. J. 25 (1945), 46156. Reprinted in: Selected Papers on Noise and Stochastic Processes (ed. by N. Wax), Dover, New York (1954), 133-294. 


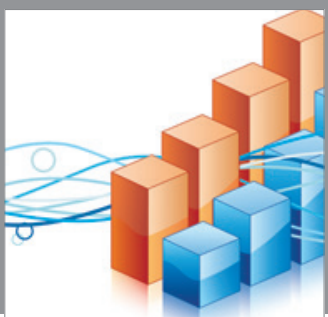

Advances in

Operations Research

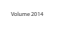

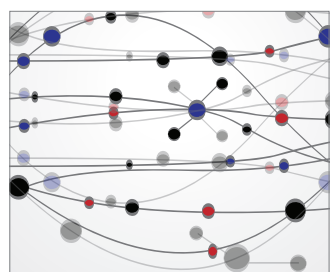

\section{The Scientific} World Journal
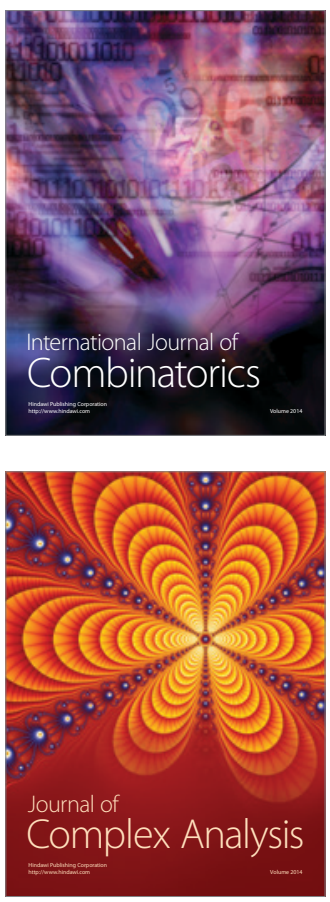

International Journal of

Mathematics and

Mathematical

Sciences
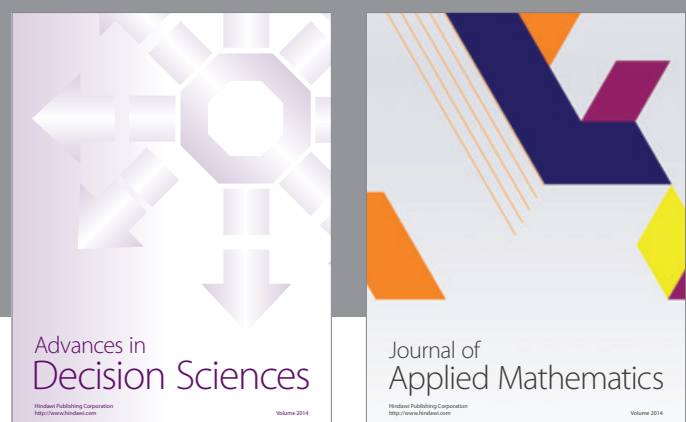

Journal of

Applied Mathematics
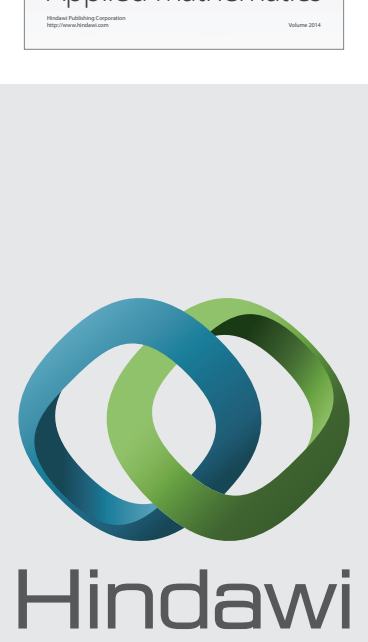

Submit your manuscripts at http://www.hindawi.com
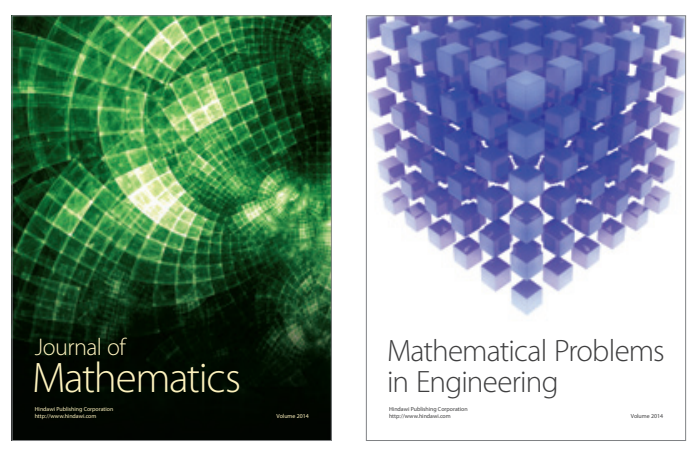

Mathematical Problems in Engineering
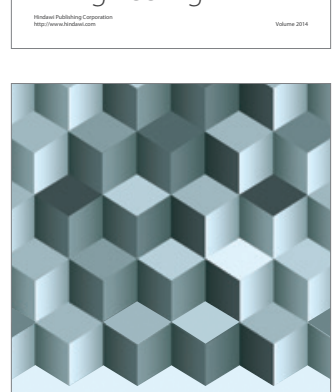

Journal of

Function Spaces
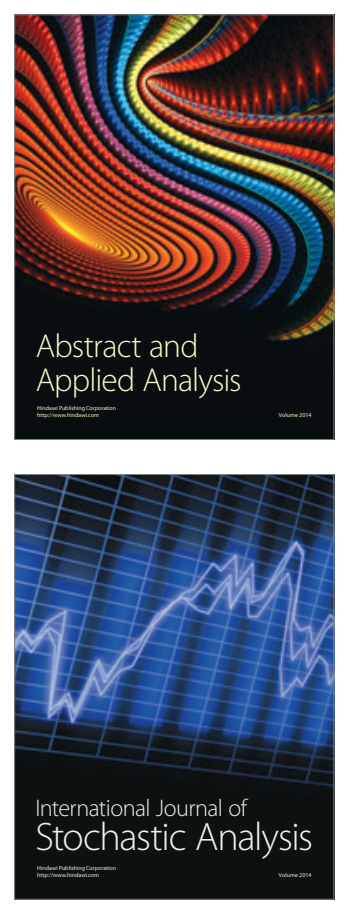

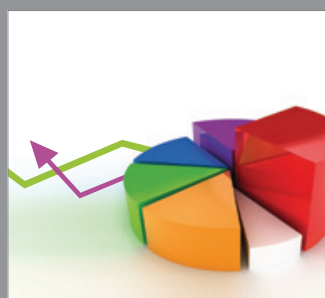

ournal of

Probability and Statistics

Promensencen
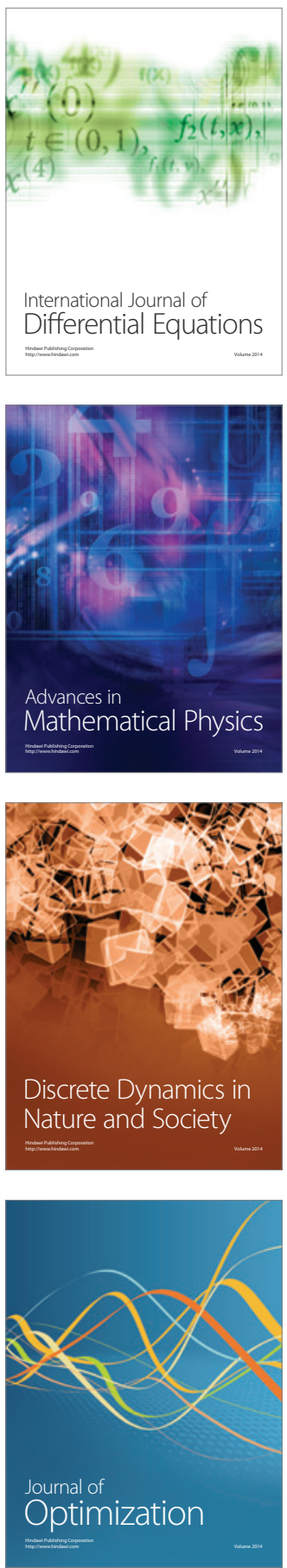